\title{
THE EQUIVALENCE OF LINEAR AND NONLINEAR DIFFERENTIAL EQUATIONS
}

\section{ROBERT TAYLOR HERBST}

1. Introduction. Following a remark by Pinney [2] that the equation $y^{\prime \prime}+q(x) y+c y^{-3}=0$ can be solved by putting $y^{2}=u^{2}-v^{2}$, where $u, v$ are appropriately chosen solutions of $Y^{\prime \prime}+q Y=0, \mathrm{~J}$. M. Thomas [3] raised the question: What equations of order $n$ have general solution expressible as $F\left(u_{1}, \cdots, u_{n}\right)$, where $u_{1}, \cdots, u_{n}$ constitute a variable set of solutions of a fixed linear differential equation?, and gave a complete answer to this question for linear equations, which are of the first order, which are homogeneous of the second order, $F$ depending on only one $u$, and which are homogeneous of the second order, $F$ homogeneous of nonzero degree in two $u$ 's.

The present paper gives the complete answer to the above question for linear homogeneous equations of the second order. In addition, new integrable types of nonlinear equations are given.

It is shown that:

If $u, v$ are variable independent solutions with Wronskian w of the linear equation

$$
Y^{\prime \prime}-w(x)^{-1} w^{\prime}(x) Y^{\prime}+q(x) Y=0,
$$

where $w$ and $q$ are given functions, then the equation

$$
y^{\prime \prime}-w^{-1} w^{\prime} y^{\prime}=f\left(y, y^{\prime}, w, q\right)
$$

has general solution

$$
y=F(u, v)
$$

if and only if

$$
f=-q Z(y)+A(y) y^{\prime 2}+w^{2} C(y),
$$

where $Z, A, C$ satisfy

$$
Z C^{\prime}+(3-A Z) C=0, \quad Z^{\prime}-A Z=1 .
$$

The $F$ in (1.3) is any solution of the system 1955.

Presented to the Society, December 27, 1954; received by the editors April 6,

1 Throughout this paper' denotes the total derivative with respect to the explicit variable. 


$$
\begin{array}{ll}
F_{u u}=A(F) F_{u}^{2}+v^{2} C(F), & F_{v v}=A(F) F_{v}^{2}+u^{2} C(F), \\
F_{u v}=A(F) F_{u} F_{v}-u v C(F), & F_{u}=u^{-1}\left(Z(F)-v F_{v}\right) .
\end{array}
$$

2. The general solution. If the system (1.6) has a solution, then the system is passive (see $[4$, p. 274$]$ for a definition of passivity). Let $F$ be a solution of (1.6), and let $y=F$ satisfy (1.2). It follows that $A, C, Z$ satisfy (1.5) and

(2.1) $u^{\prime \prime} F_{u}+v^{\prime \prime} F_{v}+u^{\prime 2} F_{u u}+2 u^{\prime} v^{\prime} F_{u v}+v^{\prime 2} F_{v v}-w^{-1} w^{\prime}\left(u^{\prime} F_{u}+v^{\prime} F_{v}\right)=f$.

When $u^{\prime \prime}, v^{\prime \prime}, u^{\prime}, v^{\prime}, F_{u u}, F_{u v}, F_{v v}, F_{u}, F_{v}$ are eliminated from (2.1) by means of (1.1) and (1.6), (1.4) results.

If $f$ is given by (1.4), and $Z, A, C$ satisfy (1.5), then the substitution $y=F$ in (1.2) gives (1.6), which by virtue of (1.5) is passive and can be integrated giving an $F$ satisfying (1.2).

If initial values $y_{0}, y_{0}^{\prime}$ are given, then from the relations [3, equation (4.1)]

$$
y=F, \quad u^{\prime} Z=u y^{\prime}-w F_{v}, \quad v^{\prime} Z=v y^{\prime}+w F_{u}
$$

a set of initial values $u_{0}, v_{0}, u_{0}^{\prime}, v_{0}^{\prime}$ can be found such that $w_{0}=u_{0} v_{0}^{\prime}$ $-u_{0}^{\prime} v_{0}$. Thus $y=F$ is the general solution of (1.2), and the result is established.

From (1.5) and (1.6), it follows that $y=F(u)$ implies $C=0$. In this case the theorem reduces to that given in $[3, \S 3]$.

3. The case $Z=0$. If $F$ is homogeneous of degree $k$ in $u, v$, then $Z=k F$. Thus $k=0$ implies $Z=0$ and $F=F\left(u^{-1} v\right)$. Thomas remarks $[3$, p. 903$]$ that in this case $F$ is a function of a single variable, but that variable does not satisfy a given linear equation, and he leaves the case $k=0$ to be settled. Since (1.6) was obtained from [3, equation (4.3) ] by inverting a matrix with determinant equal to $Z^{3}$, it is clear that the preceding method will not treat this singular case.

Put $y=F(s)$, where $s=u^{-1} v$. Then

$$
y^{\prime \prime}-w^{-1} w^{\prime} y^{\prime}=w^{2} u^{-4}\left(F^{\prime \prime}-2 w^{-1} u u^{\prime} F^{\prime}\right) \text {. }
$$

By hypothesis the right member of (3.1) is to be a function of $y, y^{\prime}$, $w, q$ alone. Hence it must not change when the initial values of $u, v$, $u^{\prime}, v^{\prime}$ are varied so that $u^{-1} v, w, u^{-2} w$ are fixed. Fix the initial values of $u, v, w$, vary that of $u^{\prime}$, and determine that of $v^{\prime}$ so that $v^{\prime}=u^{-1} w$ $+u^{-1} u^{\prime} v$ initially. If $F^{\prime} \neq 0$, the right member of (3.1) varies. If $F^{\prime}=0,(3.1)$ is linear, and $y=F$ gives only its trivial and not its general solution. Thus for $n=2$ the result in $\$ 1$ gives answers to the questions raised by Thomas. 
4. Classification of equations. One application of the foregoing is in the classification of known integrable types. The four equations given below include as special cases 43 of the nonlinear equations appearing in Kamke's Collection [1, equations 6.1-6.249]. Moreover, I-IV do not appear in Kamke's Collection in the general form given here. Indeed, new integrable types have been found.

I. $y^{\prime \prime}-w(x)^{-1} w^{\prime}(x) y^{\prime}+k q(x) y=(1-l) y^{-1} y^{\prime 2}-k / 4 w(x)^{2} y^{1-4 l} \quad[3$, equation (1.1)].

Solution: $y^{2}=u^{k} v^{k}$, where $k l=1$, and $u, v$ satisfy $Y^{\prime \prime}-w^{-1} w^{\prime} Y^{\prime}$ $+q Y=0$.

II. $y^{\prime \prime}+p(x) y^{\prime}+q(x) Z(y)=A(y) y^{\prime 2}[3$, equation (3.2)].

Solution: $y=F(u)$, where $F^{\prime \prime}=A(F) F^{\prime 2}, Z^{\prime}-A Z=1$, and $u^{\prime \prime}+p u^{\prime}$ $+q u=0$.

III. $y^{\prime \prime}+p(x) y^{\prime}+q(x) y \log y=y^{-1} y^{\prime 2}$.

Solution: $y=e^{u}$, where $u^{\prime \prime}+p u^{\prime}+q u=0$.

IV. $y^{\prime \prime}+p(x) y^{\prime \prime}+q(x)\left(k y-\beta y^{1-l}\right)=(1-l) y^{-1} y^{\prime 2}$.

Solution: $y=(u+\beta / k)^{k}$, where $k l=1$, and $u^{\prime \prime}+p u^{\prime}+q u=0$.

\section{REFERENCES}

1. E. Kamke, Differentialgleichungen: Lösungsmethoden und Lösungen, New York, 1948.

2. E. Pinney, The nonlinear differential equation $y^{\prime \prime}+p(x) y+c y^{-3}=0$, Proc. Amer. Math. Soc. vol. 1 (1950) p. 681.

3. J. M. Thomas, Equations equivalent to a linear differential equation, Proc. Amer. Math. Soc. vol. 3 (1952) pp. 899-903.

4. - Orderly differential systems, Duke Math. J. vol. 7 (1940) pp. 249-290.

Office of Ordnance Research, Department of the Army 